PROCEEDINGS OF THE

AMERICAN MATHEMATICAL SOCIETY

Volume 127, Number 8, Pages 2199-2212

S 0002-9939(99)05183-7

Article electronically published on April 8, 1999

\title{
THE MOD 2 COHOMOLOGY OF THE LINEAR GROUPS OVER THE RING OF INTEGERS
}

\author{
DOMINIQUE ARLETTAZ, MAMORU MIMURA, KOJI NAKAHATA, AND \\ NOBUAKI YAGITA \\ (Communicated by Ralph Cohen)
}

\begin{abstract}
This paper completely determines the Hopf algebra structure of the $\bmod 2$ cohomology of the linear groups $G L(\mathbb{Z}), S L(\mathbb{Z})$ and $S t(\mathbb{Z})$ as a module over the Steenrod algebra, and provides an explicit description of the generators.
\end{abstract}

\section{INTRODUCTION}

Recently, J. Rognes and C. Weibel deduced from V. Voevodsky's proof [V] of the Milnor conjecture the complete calculation of the 2-torsion of the algebraic Ktheory of the ring of integers $\mathbb{Z}$ (see Table 1 of [W] and Theorem 0.6 of [RW]). Of course, this has immediate consequences on the mod 2 cohomology of the infinite general linear group $G L(\mathbb{Z})$ and more generally on the understanding of the space $B G L(\mathbb{Z})^{+}$.

In [Bok], M. Bökstedt tried to construct a 2-adic model for the space $B G L(\mathbb{Z})^{+}$: he considered any prime number $p \equiv 3$ or $5 \bmod 8$ and introduced a space $J(p)$ which is defined by the pull-back diagram

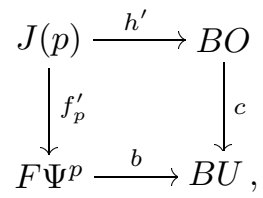

where $F \Psi^{p}$ is the fiber of $\left(\Psi^{p}-1\right): B U \rightarrow B U$ (recall that $F \Psi^{p} \simeq B G L\left(\mathbb{F}_{p}\right)^{+}$ by Theorem 7 of [Q2]), $b$ is the Brauer lifting and $c$ is the complexification. The fibers of the horizontal maps are homotopy equivalent to the unitary group $U$. He was actually more precisely interested in the covering space $J K(\mathbb{Z}, p)$ of $J(p)$ corresponding to the cyclic subgroup of order 2 of $\pi_{1} J(p) \cong \mathbb{Z} \oplus \mathbb{Z} / 2$. Bökstedt's definition of the space $J K(\mathbb{Z}, p)$ (see [Bok], Definition 1.7 and the proof of Lemma 2.1 ) is based on the Adams conjecture and on the calculation of the 2-primary part of the homotopy groups of $\left(F \Psi^{p}\right)_{2}$ which is the same, in dimensions $\equiv 3 \bmod 4$, for

Received by the editors September 15, 1997.

1991 Mathematics Subject Classification. Primary 20G10; Secondary 19D55, 20J05, 55R40, $55 \mathrm{~S} 10$.

We would like to thank Christian Ausoni for his helpful comments on Bökstedt's work [Bok] and the referee for his interesting suggestions. The third author thanks the Swiss National Science Foundation for financial support. 
all primes $p \equiv 3$ or $5 \bmod 8$ (this explains the choice of $p$; see Section 3 of $[\mathrm{Au}]$ for more details). Notice that the space $J K(\mathbb{Z}, p)$, in the case $p=3$, appears also in Section 4 of $[\mathrm{DF}]$ and in $[\mathrm{M}]$. After completion at the prime 2, Bökstedt constructed a map

$$
\varphi:\left(B G L(\mathbb{Z})^{+} \hat{)}_{2} \longrightarrow J K(\mathbb{Z}, p) \hat{)_{2}}\right.
$$

which induces a split surjection on all homotopy groups (see [Bok], Diagram 1.9). Recall that the localization exact sequence in K-theory implies that

$$
\left(B G L\left(\mathbb{Z}\left[\frac{1}{2}\right]\right)^{+}\right)_{2} \simeq\left(B G L(\mathbb{Z})^{+}\right)_{2} \times\left(S^{1} \hat{)_{2}} .\right.
$$

Therefore, $\varphi$ provides a map

$$
\widetilde{\varphi}:\left(B G L\left(\mathbb{Z}\left[\frac{1}{2}\right]\right)^{+}\right)_{2} \longrightarrow J(p) \hat{{ }_{2}}
$$

which also induces a split surjection on all homotopy groups. Bökstedt's idea was indeed excellent because now the 2-torsion of $K_{*}(\mathbb{Z})$ is known and turns out to be isomorphic to the 2-torsion of $\pi_{*} J K(\mathbb{Z}, p)$ (according to Table 1 of [W] and Theorem 0.6 of $[\mathrm{RW}])$; therefore, $\varphi$ and $\widetilde{\varphi}$ are actually homotopy equivalences. Observe in particular that the homotopy type of $\left(J K(\mathbb{Z}, p) \hat{)_{2}}\right.$ does not depend on $p$ (for $p \equiv 3$ or $5 \bmod 8$ ). Consequently, we obtain for all primes $p \equiv 3$ or $5 \bmod 8$ the pull-back diagram (see also Corollary 8 of $[\mathrm{W}]$ )

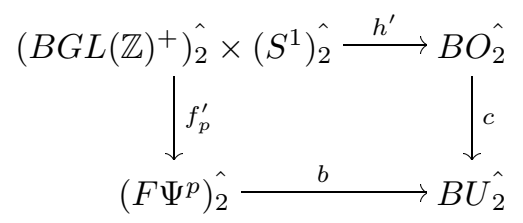

and the commutative diagram (where both rows are fibrations)

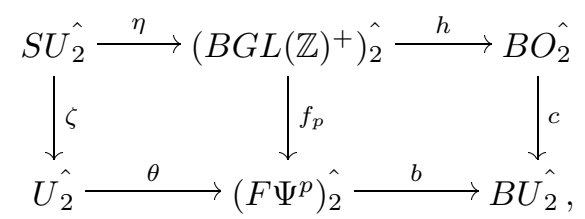

in which $f_{p}$ and $h$ denote the composition of the inclusion

$$
\left(B G L ( \mathbb { Z } ) ^ { + } \hat { ) } _ { 2 } \hookrightarrow \left(B G L(\mathbb{Z})^{+} \hat{)_{2}} \times\left(S^{1} \hat{)_{2}}\right.\right.\right.
$$

with $f_{p}^{\prime}$ and $h^{\prime}$ respectively, and $\zeta$ the 2-completion of the inclusion $S U \hookrightarrow U \simeq$ $S U \times S^{1}$. According to Section 2 of [Bok], the map $h$ is induced by the inclusion $\mathbb{Z} \hookrightarrow \mathbb{R}$ and for all odd primes $p$, the diagram

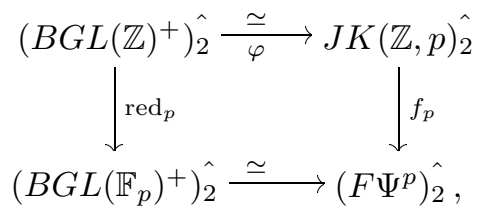

where $\operatorname{red}_{p}$ is the map induced by the reduction $\bmod p: G L(\mathbb{Z}) \rightarrow G L\left(\mathbb{F}_{p}\right)$, is homotopy commutative. Thus, we may assume that the map $f_{p}$ in the diagram (*) is induced by the reduction $\bmod p$. 
S. Mitchell computed the mod 2 homology of the space $J K(\mathbb{Z}, 3)$ in Theorem 4.3 of $[\mathrm{M}]$; because of the above homotopy equivalence $\left(B G L(\mathbb{Z})^{+}\right)_{2} \simeq J K\left(\mathbb{Z}, 3 \hat{)_{2}}\right.$, this provides the calculation of $H_{*}\left(B G L(\mathbb{Z})^{+} ; \mathbb{Z} / 2\right)$ and by dualization the determination of the Hopf algebra structure of $H^{*}\left(B G L(\mathbb{Z})^{+} ; \mathbb{Z} / 2\right)$ as a module over the Steenrod algebra $\mathcal{A}$ (see $[\mathrm{M}]$, Remark 4.5). However, Mitchell's argument does not give explicit generators of $H^{*}\left(B G L(\mathbb{Z})^{+} ; \mathbb{Z} / 2\right)$. The first goal of the present paper is to use the above commutative diagram $(*)$ in order to get a direct proof of Michell's result.

Theorem. There is an isomorphism of Hopf algebras and of modules over the Steenrod algebra

$$
\alpha: H^{*}\left(B G L(\mathbb{Z})^{+} ; \mathbb{Z} / 2\right) \cong H^{*}(B O ; \mathbb{Z} / 2) \otimes H^{*}(S U ; \mathbb{Z} / 2) .
$$

Recall that $H^{*}(B O ; \mathbb{Z} / 2) \cong \mathbb{Z} / 2\left[w_{1}, w_{2}, \ldots\right]$ and $H^{*}(S U ; \mathbb{Z} / 2) \cong \Lambda\left(v_{3}, v_{5}, \ldots\right)$, where $\operatorname{deg}\left(w_{j}\right)=j$ and $\operatorname{deg}\left(v_{2 k-1}\right)=2 k-1$.

In fact, the main objective of this paper is to describe explicitly the generators of $H^{*}\left(B G L(\mathbb{Z})^{+} ; \mathbb{Z} / 2\right)$. The generators of the polynomial part are the Stiefel-Whitney classes, also denoted by $w_{j}$, coming from $H^{*}(B O ; \mathbb{Z} / 2)$ via the homomorphism induced by $h$. On the other hand, we identify precisely (see Definitions 5 and 10 and Remark 14) the exterior generators $u_{2 k-1}$ of degree $2 k-1$ in $H^{*}\left(B G L(\mathbb{Z})^{+} ; \mathbb{Z} / 2\right)$, corresponding to $1 \otimes v_{2 k-1}$ under the above isomorphism $\alpha$, in terms of the image of the homomorphism

$$
f_{p}^{*}: H^{*}\left(F \Psi^{p} ; \mathbb{Z} / 2\right) \cong H^{*}\left(B G L\left(\mathbb{F}_{p}\right)^{+} ; \mathbb{Z} / 2\right) \rightarrow H^{*}\left(B G L(\mathbb{Z})^{+} ; \mathbb{Z} / 2\right)
$$

induced by the reduction mod $p$ for $p \equiv 5 \bmod 8$ (they actually do not depend on the choice of $p$ ). We show that the classes $u_{2 k-1}$ are primitive cohomology classes and compute the action of the Steenrod squares on them. Therefore, we get an isomorphism of Hopf algebras and of modules over the Steenrod algebra

$$
H^{*}\left(B G L(\mathbb{Z})^{+} ; \mathbb{Z} / 2\right) \cong \mathbb{Z} / 2\left[w_{1}, w_{2}, \ldots\right] \otimes \Lambda\left(u_{3}, u_{5}, \ldots\right)
$$

and we deduce that the isomorphism $\alpha$ is unique (see Theorem 11). We also obtain an explicit formula relating the classes $u_{2 k-1}$ to the image of the homomorphism $f_{p}^{*}$ for all primes $p \equiv 3 \bmod 8$ (see Theorem 13 ).

This provides a complete description of the mod 2 cohomology of the infinite general linear group $G L(\mathbb{Z})$. In the remainder of the paper we compute the mod 2 cohomology of the infinite special linear group $S L(\mathbb{Z})$ and of the infinite Steinberg group $\operatorname{St}(\mathbb{Z})$ (see Corollary 15, Theorem 17 and Remark 18).

\section{The mod 2 cohomology of the linear groups $G L(\mathbb{Z})$ and $S L(\mathbb{Z})$}

Theorem 1. There is an isomorphism of Hopf algebras and of modules over the Steenrod algebra

$$
\alpha: H^{*}\left(B G L(\mathbb{Z})^{+} ; \mathbb{Z} / 2\right) \cong H^{*}(B O ; \mathbb{Z} / 2) \otimes H^{*}(S U ; \mathbb{Z} / 2) .
$$

Proof. As mentioned in the introduction, this follows indirectly from $[\mathrm{M}]$, Theorem 4.3 and Remark 4.5. Here is a direct argument. Let $Q$ denote the subgroup of diagonal matrices in $G L(\mathbb{Z})$ and let $\lambda: B Q \rightarrow B G L(\mathbb{Z})^{+}$be the map induced by the inclusion $Q \hookrightarrow G L(\mathbb{Z})$. It is known by Theorem 22.7 of [Bor] that the composition $h \lambda: B Q \rightarrow B O$ induces an injective homomorphism $\lambda^{*} h^{*}: H^{*}(B O ; \mathbb{Z} / 2) \cong$ $\mathbb{Z} / 2\left[w_{1}, w_{2}, \ldots\right] \rightarrow H^{*}(B Q ; \mathbb{Z} / 2) \cong \lim _{m} \mathbb{Z} / 2\left[z_{1}, z_{2}, \ldots, z_{m}\right]\left(\right.$ with $\left.\operatorname{deg}\left(z_{i}\right)=1\right)$ and that $\lambda^{*} h^{*}\left(w_{j}\right)=\sigma_{j}$, where $\sigma_{j}$ is the element of $H^{j}(B Q ; \mathbb{Z} / 2)$ whose restriction 
to $\mathbb{Z} / 2\left[z_{1}, z_{2}, \ldots, z_{m}\right]$ is the $j$-th elementary symmetric function in the $m$ variables $z_{1}, \ldots, z_{m}$, for all $m \geq j$. This implies that the infinite loop map $h$ induces an injective homomorphism $h^{*}: H^{*}(B O ; \mathbb{Z} / 2) \rightarrow H^{*}\left(B G L(\mathbb{Z})^{+} ; \mathbb{Z} / 2\right)$. Therefore, Theorem 15.2 of [Bor] shows that the Serre spectral sequence of the fibration

$$
S \hat{U_{2}} \stackrel{\eta}{\longrightarrow}\left(B G L(\mathbb{Z})^{+} \hat{)_{2}} \stackrel{h}{\longrightarrow} B \hat{O_{2}}\right.
$$

collapses (see also Corollary 4.3 of $[\mathrm{DF}]$ ) and we get additively the desired isomorphism. Since $\left(B G L(\mathbb{Z})^{+}\right)_{2}$ is an H-space, the maps $\lambda$ and $\eta$ produce an H-map

$$
\psi: B Q \times S \hat{U_{2}} \longrightarrow\left(B G L(\mathbb{Z})^{+}\right)_{2}
$$

which induces an injective $\mathcal{A}$-module Hopf algebra homomorphism

$$
\psi^{*}: H^{*}\left(B G L(\mathbb{Z})^{+} ; \mathbb{Z} / 2\right) \longrightarrow H^{*}(B Q ; \mathbb{Z} / 2) \otimes H^{*}(S U ; \mathbb{Z} / 2) .
$$

Moreover, the fact that $\lambda^{*}: H^{*}\left(B G L(\mathbb{Z})^{+} ; \mathbb{Z} / 2\right) \rightarrow H^{*}(B Q ; \mathbb{Z} / 2)$ also satisfies $\lambda^{*}\left(w_{j}\right)=\sigma_{j}$ (see Lemma 1.1 of [Ar1]) implies that the image of $\psi^{*}$ is isomorphic to $R \otimes H^{*}(S U ; \mathbb{Z} / 2)$, where $R$ is the subalgebra of $H^{*}(B Q ; \mathbb{Z} / 2)$ generated by the elementary symmetric functions $\sigma_{j}$. On the other hand, the image of the injective $\mathcal{A}$-module Hopf algebra homomorphism

$$
\lambda^{*} h^{*} \otimes 1: H^{*}(B O ; \mathbb{Z} / 2) \otimes H^{*}(S U ; \mathbb{Z} / 2) \longrightarrow H^{*}(B Q ; \mathbb{Z} / 2) \otimes H^{*}(S U ; \mathbb{Z} / 2)
$$

is also $R \otimes H^{*}(S U ; \mathbb{Z} / 2)$. This provides the statement of the theorem.

In order to get a more precise picture of $H^{*}\left(B G L(\mathbb{Z})^{+} ; \mathbb{Z} / 2\right)$, let us identify its generators and understand the action of the Steenrod algebra on them. For $j \geq 1$ let us write $w_{j} \in H^{*}\left(B G L(\mathbb{Z})^{+} ; \mathbb{Z} / 2\right)$ for the image of the $j$-th universal StiefelWhitney class in $H^{*}(B O ; \mathbb{Z} / 2)$ under the homomorphism $h^{*}: H^{*}(B O ; \mathbb{Z} / 2) \rightarrow$ $H^{*}\left(B G L(\mathbb{Z})^{+} ; \mathbb{Z} / 2\right)$. The action of the Steenrod algebra on the Stiefel-Whitney classes is known by Wu's formula (see for instance [MT], Part I, p. 141). It remains to identify the exterior generators of $H^{*}\left(B G L(\mathbb{Z})^{+} ; \mathbb{Z} / 2\right)$. This will be done by using the homomorphism $f_{p}^{*}: H^{*}\left(F \Psi^{p} ; \mathbb{Z} / 2\right) \rightarrow H^{*}\left(B G L(\mathbb{Z})^{+} ; \mathbb{Z} / 2\right)$ induced by the map $f_{p}$.

Let us first recall some properties of $H^{*}\left(F \Psi^{p} ; \mathbb{Z} / 2\right) \cong H^{*}\left(B G L\left(\mathbb{F}_{p}\right)^{+} ; \mathbb{Z} / 2\right)$. According to Quillen's calculation and notation (see [Q2]), if $p$ is a prime $\equiv 5 \bmod 8$, then

$$
H^{*}\left(F \Psi^{p} ; \mathbb{Z} / 2\right) \cong \mathbb{Z} / 2\left[c_{1}, c_{2}, \ldots\right] \otimes \Lambda\left(e_{1}, e_{2}, \ldots\right),
$$

where $\operatorname{deg} c_{j}=2 j$ and $\operatorname{deg} e_{k}=2 k-1$; if $p$ is a prime $\equiv 3 \bmod 8$, then $H^{*}\left(F \Psi^{p} ; \mathbb{Z} / 2\right)$ is also generated by the classes $c_{j}$ and $e_{k}(j \geq 1, k \geq 1)$, but one has the relations

$$
e_{k}^{2}=c_{2 k-1}+\sum_{j=1}^{k-1} c_{j} c_{2 k-1-j}
$$

for $k \geq 1$, and $H^{*}\left(F \Psi^{p} ; \mathbb{Z} / 2\right)$ is polynomial:

$$
H^{*}\left(F \Psi^{p} ; \mathbb{Z} / 2\right) \cong \mathbb{Z} / 2\left[e_{1}, e_{2}, \ldots, c_{2}, c_{4}, \ldots\right]
$$

(see also Section IV.8 of [FP]). In both cases, $c_{j}$ is the image under $b^{*}: H^{*}(B U ; \mathbb{Z} / 2)$ $\rightarrow H^{*}\left(F \Psi^{p} ; \mathbb{Z} / 2\right)$ of the reduction $\bmod 2$ of the $j$-th universal Chern class in $H^{2 j}(B U ; \mathbb{Z})$ and a spectral sequence argument shows that

$$
\theta^{*}: H^{*}\left(F \Psi^{p} ; \mathbb{Z} / 2\right) \rightarrow H^{*}(U ; \mathbb{Z} / 2) \cong \Lambda\left(v_{1}, v_{2}, \ldots\right)
$$


satisfies $\theta^{*}\left(e_{k}\right)=v_{2 k-1}$ for $k \geq 1$. For a prime $p \equiv 3$ or $5 \bmod 8$, consider the homomorphism $f_{p}^{*}: H^{*}\left(F \Psi^{p} ; \mathbb{Z} / 2\right) \rightarrow H^{*}\left(B G L(\mathbb{Z})^{+} ; \mathbb{Z} / 2\right)$ induced by $f_{p}$. For all $j \geq 1$, it is well known (see also Lemma 1.4 of $[\mathrm{Ar} 1]$ ) that

$$
f_{p}^{*}\left(c_{j}\right)=w_{j}^{2}
$$

and we established in [Ar2] for $k \geq 2$ the nonvanishing of the exterior class $f_{p}^{*}\left(e_{k}\right)$ if $p \equiv 5 \bmod 8$, respectively of the exterior class

$$
\gamma_{k}=f_{p}^{*}\left(e_{k}\right)+w_{2 k-1}+\sum_{j=1}^{k-1} w_{j} w_{2 k-j-1}
$$

of degree $2 k-1$ if $p \equiv 3 \bmod 8$.

Let us mention the effect of the Steenrod squares on these cohomology classes.

Lemma 2. (a) In $H^{*}(S U ; \mathbb{Z} / 2), S q^{2 i} v_{2 k-1}=\left(\begin{array}{c}k-1 \\ i\end{array}\right) v_{2 k+2 i-1}$ for $k \geq 2,1 \leq i<k$, and $S q^{2 i-1} v_{2 k-1}=0$ for $k \geq 2,1 \leq i \leq k$.

(b) In $H^{*}\left(B G L(\mathbb{Z})^{+} ; \mathbb{Z} / 2\right)$, for any odd prime $p$, for $k \geq 1$ and $1 \leq i<k$,

$$
S q^{2 i} f_{p}^{*}\left(e_{k}\right)=\left(\begin{array}{c}
k-1 \\
i
\end{array}\right) f_{p}^{*}\left(e_{k+i}\right)+\sum_{j=1}^{i}\left(\begin{array}{c}
k-j-1 \\
i-j
\end{array}\right)\left(w_{j}^{2} f_{p}^{*}\left(e_{k+i-j}\right)+w_{k+i-j}^{2} f_{p}^{*}\left(e_{j}\right)\right) .
$$

(c) In $H^{*}\left(B G L(\mathbb{Z})^{+} ; \mathbb{Z} / 2\right)$, for $k \geq 1$ and $1 \leq i \leq k$,

$$
S q^{2 i-1} f_{p}^{*}\left(e_{k}\right)=\left\{\begin{array}{l}
0, \quad \text { if } p \equiv 1 \bmod 4 \text { or if } p \equiv 3 \bmod 4 \text { and } k-i \text { is odd, } \\
\sum_{j=0}^{i-1}\left(\begin{array}{c}
k-j-1 \\
i-j-1
\end{array}\right) w_{j}^{2} w_{k+i-j-1}^{2}, \quad \text { if } p \equiv 3 \bmod 4 \text { and } k-i \text { is even. }
\end{array}\right.
$$

(d) In $H^{*}\left(B G L(\mathbb{Z})^{+} ; \mathbb{Z} / 2\right)$, for any prime $p \equiv 3 \bmod 8$ and for $k \geq 1$,

$$
S q^{2 i} \gamma_{k}=\left(\begin{array}{c}
k-1 \\
i
\end{array}\right) \gamma_{k+i}+\sum_{j=1}^{i}\left(\begin{array}{c}
k-j-1 \\
i-j
\end{array}\right)\left(w_{j}^{2} \gamma_{k+i-j}+w_{k+i-j}^{2} \gamma_{j}\right)
$$

for $1 \leq i<k$ and $S q^{2 i-1} \gamma_{k}=0$ for $1 \leq i \leq k$.

Proof. Lemma 4 of [Ar2] gives the following information on the action of the Steenrod squares on the classes $e_{k} \in H^{*}\left(F \Psi^{p} ; \mathbb{Z} / 2\right)$ for $k \geq 1$ : for any odd prime $p$ and for $1 \leq i<k$,

$$
S q^{2 i} e_{k}=\left(\begin{array}{c}
k-1 \\
i
\end{array}\right) e_{k+i}+\sum_{j=1}^{i}\left(\begin{array}{c}
k-j-1 \\
i-j
\end{array}\right)\left(c_{j} e_{k+i-j}+c_{k+i-j} e_{j}\right),
$$

and for $1 \leq i \leq k$,

$$
S q^{2 i-1} e_{k}=\left\{\begin{array}{l}
0, \quad \text { if } p \equiv 1 \bmod 4 \text { or if } p \equiv 3 \bmod 4 \text { and } k-i \text { is odd, } \\
\sum_{j=0}^{i-1}\left(\begin{array}{c}
k-j-1 \\
i-j-1
\end{array}\right) c_{j} c_{k+i-j-1}, \quad \text { if } p \equiv 3 \bmod 4 \text { and } k-i \text { is even. }
\end{array}\right.
$$

The formula (a) is well known but can be deduced from the previous equalities because the composition $\zeta^{*} \theta^{*}: H^{*}\left(F \Psi^{p} ; \mathbb{Z} / 2\right) \rightarrow H^{*}(S U ; \mathbb{Z} / 2)$ satisfies $\zeta^{*} \theta^{*}\left(e_{k}\right)=$ $v_{2 k-1}$ for $k \geq 2$ and $\zeta^{*} \theta^{*}\left(c_{j}\right)=0$ for $j \geq 1$. The statements (b) and (c) follow directly since $S q^{2 i} f_{p}^{*}\left(e_{k}\right)=f_{p}^{*}\left(S q^{2 i} e_{k}\right)$ and $f_{p}^{*}\left(c_{j}\right)=w_{j}^{2}$ for $j \geq 1$. In order to get $(\mathrm{d})$, let us consider again the homomorphism $\lambda^{*}: H^{*}\left(B G L(\mathbb{Z})^{+} ; \mathbb{Z} / 2\right) \rightarrow$ 
$H^{*}(B Q ; \mathbb{Z} / 2)$ which is injective on $\mathbb{Z} / 2\left[w_{1}, w_{2}, \ldots\right]$ and trivial on exterior classes because $H^{*}(B Q ; \mathbb{Z} / 2)$ is polynomial. If $p \equiv 3 \bmod 8$, one has by the definition of $\gamma_{k}$

$$
S q^{2 i} \gamma_{k}=S q^{2 i} f_{p}^{*}\left(e_{k}\right)+S q^{2 i} w_{2 k-1}+\sum_{j=1}^{k-1} S q^{2 i}\left(w_{j} w_{2 k-j-1}\right)
$$

for $1 \leq i<k$. According to (b),

$$
\begin{aligned}
S q^{2 i} \gamma_{k}= & \left(\begin{array}{c}
k-1 \\
i
\end{array}\right) f_{p}^{*}\left(e_{k+i}\right)+\sum_{j=1}^{i}\left(\begin{array}{c}
k-j-1 \\
i-j
\end{array}\right)\left(w_{j}^{2} f_{p}^{*}\left(e_{k+i-j}\right)+w_{k+i-j}^{2} f_{p}^{*}\left(e_{j}\right)\right) \\
& +\left(\text { element of } \mathbb{Z} / 2\left[w_{1}, w_{2}, \ldots\right]\right)
\end{aligned}
$$

and consequently,

$$
\begin{aligned}
S q^{2 i} \gamma_{k}= & \left(\begin{array}{c}
k-1 \\
i
\end{array}\right) \gamma_{k+i}+\sum_{j=1}^{i}\left(\begin{array}{c}
k-j-1 \\
i-j
\end{array}\right)\left(w_{j}^{2} \gamma_{k+i-j}+w_{k+i-j}^{2} \gamma_{j}\right) \\
& +\left(\text { element of } \mathbb{Z} / 2\left[w_{1}, w_{2}, \ldots\right]\right) .
\end{aligned}
$$

Since the classes $\gamma_{k}$ are exterior, they belong to the kernel of $\lambda^{*}$ and $\lambda^{*}\left(S q^{2 i} \gamma_{k}\right)=0$. However, the injectivity of $\lambda^{*}$ on Stiefel-Whitney classes implies that the element of $\mathbb{Z} / 2\left[w_{1}, w_{2}, \ldots\right]$ in the last formula vanishes. The assertion (c) shows that $S q^{2 i-1} \gamma_{k}$ is an element of $\mathbb{Z} / 2\left[w_{1}, w_{2}, \ldots\right]$ and one deduces similarly that $S q^{2 i-1} \gamma_{k}=0$.

Our argument will be based on the understanding of the homomorphism

$$
\begin{aligned}
\mu^{*}: & H^{*}\left(B G L(\mathbb{Z})^{+} ; \mathbb{Z} / 2\right) \rightarrow H^{*}\left(B G L(\mathbb{Z})^{+} \times B G L(\mathbb{Z})^{+} ; \mathbb{Z} / 2\right) \\
& \cong H^{*}\left(B G L(\mathbb{Z})^{+} ; \mathbb{Z} / 2\right) \otimes H^{*}\left(B G L(\mathbb{Z})^{+} ; \mathbb{Z} / 2\right)
\end{aligned}
$$

induced by the $\mathrm{H}$-space structure $\mu$ of $B G L(\mathbb{Z})^{+}$.

Lemma 3. (a) For any $j \geq 1$,

$$
\mu^{*}\left(w_{j}\right)=\sum_{s=0}^{j} w_{s} \otimes w_{j-s} .
$$

(b) For any prime $p \equiv 5 \bmod 8$ and any integer $k \geq 2$,

$$
\mu^{*}\left(f_{p}^{*}\left(e_{k}\right)\right)=f_{p}^{*}\left(e_{k}\right) \otimes 1+1 \otimes f_{p}^{*}\left(e_{k}\right)+\sum_{\ell=1}^{k-2}\left(w_{\ell}^{2} \otimes f_{p}^{*}\left(e_{k-\ell}\right)+f_{p}^{*}\left(e_{k-\ell}\right) \otimes w_{\ell}^{2}\right) .
$$

(c) For any prime $p \equiv 3 \bmod 8$ and any integer $k \geq 2$,

$$
\mu^{*}\left(\gamma_{k}\right)=\gamma_{k} \otimes 1+1 \otimes \gamma_{k}+\sum_{\ell=1}^{k-2}\left(w_{\ell}^{2} \otimes \gamma_{k-\ell}+\gamma_{k-\ell} \otimes w_{\ell}^{2}\right) .
$$

Proof. Assertion (a) is known (see for instance [MT], Part I, p. 140). If $\nu$ denotes the H-space structure of $F \Psi^{p}$, Proposition 2 of [Q2] implies that

$$
\begin{aligned}
\mu^{*}\left(f_{p}^{*}\left(e_{k}\right)\right) & =f_{p}^{*}\left(\nu^{*}\left(e_{k}\right)\right)=f_{p}^{*}\left(\sum_{\ell=0}^{k}\left(c_{\ell} \otimes e_{k-\ell}+e_{k-\ell} \otimes c_{\ell}\right)\right) \\
& =\sum_{\ell=0}^{k}\left(w_{\ell}^{2} \otimes f_{p}^{*}\left(e_{k-\ell}\right)+f_{p}^{*}\left(e_{k-\ell}\right) \otimes w_{\ell}^{2}\right)
\end{aligned}
$$


for any odd prime $p$. If $p \equiv 5 \bmod 8, f_{p}^{*}\left(e_{1}\right)$ vanishes since $e_{1}$ is exterior and one gets immediately (b). If $p \equiv 3 \bmod 8$, the definition of $\gamma_{k}$,

$$
\gamma_{k}=f_{p}^{*}\left(e_{k}\right)+w_{2 k-1}+\sum_{j=1}^{k-1} w_{j} w_{2 k-j-1},
$$

shows that

$$
\mu^{*}\left(\gamma_{k}\right)=\sum_{\ell=0}^{k}\left(w_{\ell}^{2} \otimes f_{p}^{*}\left(e_{k-\ell}\right)+f_{p}^{*}\left(e_{k-\ell}\right) \otimes w_{\ell}^{2}\right)+\left(\text { element of } \mathbb{Z} / 2\left[w_{1}, w_{2}, \ldots\right]\right) .
$$

Since $p \equiv 3 \bmod 8$, it turns out that $f_{p}^{*}\left(e_{1}\right)=w_{1}$ and consequently that

$$
\begin{aligned}
\mu^{*}\left(\gamma_{k}\right)=\gamma_{k} \otimes 1+1 \otimes \gamma_{k}+\sum_{\ell=1}^{k-2}\left(w_{\ell}^{2} \otimes \gamma_{k-\ell}+\gamma_{k-\ell} \otimes w_{\ell}^{2}\right) \\
+\left(\text { element of } \mathbb{Z} / 2\left[w_{1}, w_{2}, \ldots\right]\right) .
\end{aligned}
$$

However, the element of $\mathbb{Z} / 2\left[w_{1}, w_{2}, \ldots\right]$ in that formula must be trivial since $\mu^{*}\left(\gamma_{k}\right)$ is exterior. This implies the last assertion.

Now, let $p$ be a prime $\equiv 5 \bmod 8$ and $k$ an integer $\geq 2$. Consider an integer $m \geq k, C$ the cyclic group of order $p-1$ and

$$
H^{*}\left(B C^{m} ; \mathbb{Z} / 2\right) \cong \mathbb{Z} / 2\left[x_{1}, x_{2}, \ldots, x_{m}\right] \otimes \Lambda\left(y_{1}, y_{2}, \ldots, y_{m}\right)
$$

with $\operatorname{deg}\left(x_{i}\right)=2$ and $\operatorname{deg}\left(y_{i}\right)=1$ for $1 \leq i \leq m$, endowed with the differential $d$ defined by $d\left(x_{i}\right)=y_{i}$ and $d\left(y_{i}\right)=0$. Then, look at the homomorphism $\rho$ : $H^{*}\left(F \Psi^{p} ; \mathbb{Z} / 2\right) \rightarrow H^{*}\left(B C^{m} ; \mathbb{Z} / 2\right)$, introduced in [Q2], p. 563-565, which is injective in dimensions $\leq 2 m$ (and in particular in dimensions $\leq 2 k$ ) since its kernel is the ideal generated by the elements $c_{j}$ and $e_{j}$ for $j>m$, and which fulfills $\rho\left(c_{j}\right)=s_{j}$ and $\rho\left(e_{j}\right)=d\left(s_{j}\right)$ for $1 \leq j \leq m$, where $s_{j}$ denotes the $j$-th elementary symmetric function in $x_{1}, x_{2}, \ldots, x_{m}$. For $k \geq 1$, define the exterior class

$$
\xi_{k}=\sum_{j=1}^{m} x_{j}^{k-1} y_{j} \in H^{2 k-1}\left(B C^{m} ; \mathbb{Z} / 2\right) .
$$

Since $s_{k}=\sum_{i_{1}<i_{2}<\cdots<i_{k}} x_{i_{1}} x_{i_{2}} \cdots x_{i_{k}}$, one has

$$
d\left(s_{k}\right)=\sum_{i_{1}<i_{2}<\cdots<i_{k}} \sum_{\ell} x_{i_{1}} \cdots \widehat{x_{i_{\ell}}} \cdots x_{i_{k}} y_{i_{\ell}} .
$$

Then, consider the difference

$$
\begin{aligned}
d\left(s_{k}\right)-s_{k-1} \xi_{1} & =\sum_{i_{1}<i_{2}<\cdots<i_{k}} \sum_{\ell} x_{i_{1}} \cdots \widehat{x_{i_{\ell}}} \cdots x_{i_{k}} y_{i_{\ell}} \\
& -\sum_{i_{1}<i_{2}<\cdots<i_{k-1}} x_{i_{1}} x_{i_{2}} \cdots x_{i_{k-1}} \sum_{i} y_{i} \\
& =\sum_{i_{1}<i_{2}<\cdots<i_{k-1}} \sum_{\ell} x_{i_{1}} x_{i_{2}} \cdots x_{i_{\ell}} \cdots x_{i_{k-1}} y_{i_{\ell}} \\
& =\sum_{i_{1}<i_{2}<\cdots<i_{k-1}} \sum_{\ell} x_{i_{1}} x_{i_{2}} \cdots \widehat{x_{i_{\ell}}} \cdots x_{i_{k-1}} x_{i_{\ell}} y_{i_{\ell}} .
\end{aligned}
$$


From this formula, one may compute the difference

$$
d\left(s_{k}\right)-s_{k-1} \xi_{1}-s_{k-2} \xi_{2}=\sum_{i_{1}<i_{2}<\cdots<i_{k-2}} \sum_{\ell} x_{i_{1}} x_{i_{2}} \cdots \widehat{x_{\ell}} \cdots x_{i_{k-2}} x_{i_{\ell}}^{2} y_{i_{\ell}}
$$

and obtain by induction

$$
d\left(s_{k}\right)=\xi_{k}+\sum_{j=1}^{k-1} s_{j} \xi_{k-j}
$$

for $k \geq 2$. Since $\rho\left(e_{k}\right)=d\left(s_{k}\right)$ and $\rho\left(c_{j}\right)=s_{j}$, we get

$$
\rho\left(e_{k}\right)=\xi_{k}+\sum_{j=1}^{k-1} \rho\left(c_{j}\right) \xi_{k-j} .
$$

This implies inductively that the exterior class $\xi_{k}$ belongs to the image of $\rho$ and the injectivity of $\rho$ in dimensions $\leq 2 k$ produces the following lemma.

Lemma 4. For $p \equiv 5 \bmod 8$ and for any $k \geq 2$, the class $e_{k} \in H^{2 k-1}\left(F \Psi^{p} ; \mathbb{Z} / 2\right)$ satisfies

$$
e_{k}=\rho^{-1}\left(\xi_{k}\right)+\sum_{j=1}^{k-1} c_{j} \rho^{-1}\left(\xi_{k-j}\right) .
$$

Definition 5. Let $p$ be a prime $\equiv 5 \bmod 8$. For all integers $k \geq 2$, let us define the exterior class $u_{2 k-1}(p)=f_{p}^{*}\left(\rho^{-1}\left(\xi_{k}\right)\right) \in H^{2 k-1}\left(B G L(\mathbb{Z})^{+} ; \mathbb{Z} / 2\right)$, where $f_{p}^{*}$ denotes the homomorphism $H^{*}\left(F \Psi^{p} ; \mathbb{Z} / 2\right) \rightarrow H^{*}\left(B G L(\mathbb{Z})^{+} ; \mathbb{Z} / 2\right)$ induced by $f_{p}$. Observe that this definition does not depend on the choice of $m \geq k$. Notice also that $f_{p}^{*}\left(\rho^{-1}\left(\xi_{1}\right)\right)=f_{p}^{*}\left(e_{1}\right)=0$.

Proposition 6. For any prime $p \equiv 5 \bmod 8$ and for $k \geq 2$, one has:

(a) $u_{2 k-1}(p)=f_{p}^{*}\left(e_{k}\right)+\sum_{j=1}^{k-2} w_{j}^{2} u_{2 k-2 j-1}(p)$,

(b) the homomorphism $\eta^{*}: H^{*}\left(B G L(\mathbb{Z})^{+} ; \mathbb{Z} / 2\right) \rightarrow H^{*}(S U ; \mathbb{Z} / 2)$ fulfills $\eta^{*}\left(u_{2 k-1}(p)\right)=v_{2 k-1}$.

Proof. Lemma 4 implies that

$$
f_{p}^{*}\left(e_{k}\right)=u_{2 k-1}(p)+\sum_{j=1}^{k-2} w_{j}^{2} u_{2 k-2 j-1}(p)
$$

since $f_{p}^{*}\left(\rho^{-1}\left(\xi_{1}\right)\right)=0$. Consequently, (b) follows directly from the commutativity of the following diagram induced by the diagram $(*)$ of the introduction

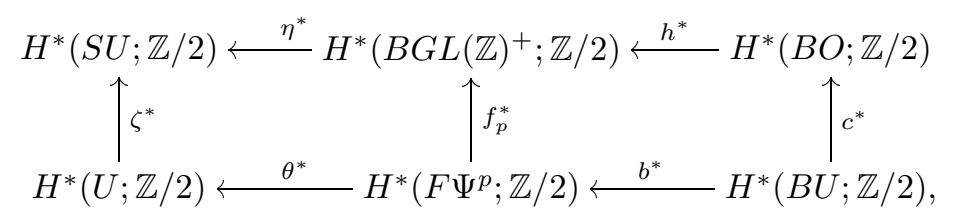

because $\eta^{*} f_{p}^{*}\left(e_{k}\right)=\zeta^{*} \theta^{*}\left(e_{k}\right)=v_{2 k-1}$ and $\eta^{*}\left(w_{j}\right)=0$ for all $k \geq 2, j \geq 1$.

Proposition 7. For any prime $p \equiv 5 \bmod 8$ and for any integer $k \geq 2$, the element $u_{2 k-1}(p)$ is a primitive cohomology class in $H^{2 k-1}\left(B G L(\mathbb{Z})^{+} ; \mathbb{Z} / 2\right)$. 
Proof. We must show that the homomorphism

$$
\mu^{*}: H^{*}\left(B G L(\mathbb{Z})^{+} ; \mathbb{Z} / 2\right) \rightarrow H^{*}\left(B G L(\mathbb{Z})^{+} \times B G L(\mathbb{Z})^{+} ; \mathbb{Z} / 2\right)
$$

satisfies $\mu^{*}\left(u_{2 k-1}(p)\right)=u_{2 k-1}(p) \otimes 1+1 \otimes u_{2 k-1}(p)$. We proceed by induction on $k$. We just established in Proposition 6 that

$$
u_{2 k-1}(p)=f_{p}^{*}\left(e_{k}\right)+\sum_{j=1}^{k-2} w_{j}^{2} u_{2 k-2 j-1}(p) .
$$

For instance, $u_{3}(p)=f_{p}^{*}\left(e_{2}\right)$ and it follows from Lemma $3(\mathrm{~b})$ that $\mu^{*}\left(u_{3}(p)\right)=$ $u_{3}(p) \otimes 1+1 \otimes u_{3}(p)$. We then may deduce from Lemma 3 (a) and (b) and the induction hypothesis that

$$
\begin{array}{r}
\mu^{*}\left(u_{2 k-1}(p)\right)=f_{p}^{*}\left(e_{k}\right) \otimes 1+1 \otimes f_{p}^{*}\left(e_{k}\right)+\sum_{\ell=1}^{k-2}\left(w_{\ell}^{2} \otimes f_{p}^{*}\left(e_{k-\ell}\right)+f_{p}^{*}\left(e_{k-\ell}\right) \otimes w_{\ell}^{2}\right) \\
+\sum_{j=1}^{k-2}\left(\sum_{s=0}^{j} w_{s}^{2} \otimes w_{j-s}^{2}\right)\left(u_{2 k-2 j-1}(p) \otimes 1+1 \otimes u_{2 k-2 j-1}(p)\right)
\end{array}
$$

and therefore that

$$
\begin{aligned}
\mu^{*}\left(u_{2 k-1}(p)\right)= & u_{2 k-1}(p) \otimes 1+1 \otimes u_{2 k-1}(p) \\
& +\sum_{\ell=1}^{k-2}\left(w_{\ell}^{2} \otimes u_{2 k-2 \ell-1}(p)+u_{2 k-2 \ell-1}(p) \otimes w_{\ell}^{2}\right) \\
& +\sum_{\ell=1}^{k-3} \sum_{t=1}^{k-\ell-2}\left(w_{\ell}^{2} \otimes w_{t}^{2} u_{2 k-2 \ell-2 t-1}(p)+w_{t}^{2} u_{2 k-2 \ell-2 t-1}(p) \otimes w_{\ell}^{2}\right) \\
& +\sum_{j=1}^{k-2}\left(w_{j}^{2} \otimes u_{2 k-2 j-1}(p)+u_{2 k-2 j-1}(p) \otimes w_{j}^{2}\right) \\
& +\sum_{j=1}^{k-2} \sum_{s=1}^{j-1}\left(w_{s}^{2} u_{2 k-2 j-1}(p) \otimes w_{j-s}^{2}+w_{s}^{2} \otimes w_{j-s}^{2} u_{2 k-2 j-1}(p)\right) .
\end{aligned}
$$

The last sum can be written as follows:

$$
\begin{aligned}
& \sum_{j=1}^{k-2} \sum_{s=1}^{j-1}\left(w_{s}^{2} u_{2 k-2 j-1}(p) \otimes w_{j-s}^{2}+w_{s}^{2} \otimes w_{j-s}^{2} u_{2 k-2 j-1}(p)\right) \\
= & \sum_{j=1}^{k-2} \sum_{s=1}^{j-1}\left(w_{s}^{2} \otimes w_{j-s}^{2} u_{2 k-2 j-1}(p)+w_{j-s}^{2} u_{2 k-2 j-1}(p) \otimes w_{s}^{2}\right) \\
= & \sum_{s=1}^{k-3} \sum_{t=1}^{k-s-1}\left(w_{s}^{2} \otimes w_{t}^{2} u_{2 k-2 s-2 t-1}(p)+w_{t}^{2} u_{2 k-2 s-2 t-1}(p) \otimes w_{s}^{2}\right) .
\end{aligned}
$$

Consequently, $\mu^{*}\left(u_{2 k-1}(p)\right)=u_{2 k-1}(p) \otimes 1+1 \otimes u_{2 k-1}(p)$ and $u_{2 k-1}(p)$ is primitive.

Remark 8. Since we know that the Hopf algebra structure of $H^{*}\left(B G L(\mathbb{Z})^{+} ; \mathbb{Z} / 2\right)$ by Theorem 1 (or $[\mathrm{M}]$, Theorem 4.3 and Remark 4.5), it is obvious that there is exactly one nontrivial primitive exterior class in each odd degree $\geq 3$ of $H^{*}\left(B G L(\mathbb{Z})^{+} ; \mathbb{Z} / 2\right)$. However, let us show it again by a computational argument. 
Lemma 9. Consider the homomorphism $\eta^{*}: H^{*}\left(B G L(\mathbb{Z})^{+} ; \mathbb{Z} / 2\right) \rightarrow H^{*}(S U ; \mathbb{Z} / 2)$. For $k \geq 2$, let $u_{2 k-1}^{\prime}$ and $u_{2 k-1}^{\prime \prime}$ be primitive exterior classes of degree $2 k-1$ in $H^{*}\left(B G L(\mathbb{Z})^{+} ; \mathbb{Z} / 2\right)$ such that $\eta^{*}\left(u_{2 k-1}^{\prime}\right)=\eta^{*}\left(u_{2 k-1}^{\prime \prime}\right)=v_{2 k-1}$. Then $u_{2 k-1}^{\prime}=$ $u_{2 k-1}^{\prime \prime}$.

Proof. Observe first that $u_{3}^{\prime}=u_{3}^{\prime \prime}$ since there is only one exterior class of degree 3 in $H^{*}\left(B G L(\mathbb{Z})^{+} ; \mathbb{Z} / 2\right)$. Then, let us define $\widetilde{u}_{2 k-1}=u_{2 k-1}^{\prime}-u_{2 k-1}^{\prime \prime}$ for all $k \geq 2$ and prove by induction on $k$ that $\widetilde{u}_{2 k-1}=0$. Since $\widetilde{u}_{2 k-1}$ is exterior and belongs to the kernel of $\eta^{*}$, the induction hypothesis shows that one can write

$$
\widetilde{u}_{2 k-1}=\sum_{s=3}^{2 k-2} u^{\prime}(s) w(s),
$$

where $u^{\prime}(s)$ is an element of degree $s$ in $\Lambda\left(u_{3}^{\prime}, u_{5}^{\prime}, \ldots, u_{2 k-3}^{\prime}\right)$ and $w(s)$ is an element of degree $2 k-s-1$ in $\mathbb{Z} / 2\left[w_{1}, w_{2}, \ldots\right]$. However, the primitivity of the classes $u_{2 j-1}^{\prime}$ and Lemma 3 (a) provide an explicit computation of $\mu^{*}\left(\widetilde{u}_{2 k-1}\right)$ which contradicts the primitivity of $\widetilde{u}_{2 k-1}$ unless one has $\widetilde{u}_{2 k-1}=0$.

Thus, we are finally able to define the exterior generators of $H^{*}\left(B G L(\mathbb{Z})^{+} ; \mathbb{Z} / 2\right)$ (see also Remark 14 below).

Definition 10. Because of Proposition 7 and Remark 8, we may conclude that the classes $u_{2 k-1}(p)$ do not depend on $p$. Therefore, for $k \geq 2$, we can define $u_{2 k-1}=u_{2 k-1}(p) \in H^{2 k-1}\left(B G L(\mathbb{Z})^{+} ; \mathbb{Z} / 2\right)$ for any prime $p \equiv 5 \bmod 8$. Since the image of $u_{2 k-1}$ under $\eta^{*}$ is $v_{2 k-1}$, the classes $u_{2 k-1}$ are nontrivial algebraically independent exterior classes in $H^{*}\left(B G L(\mathbb{Z})^{+} ; \mathbb{Z} / 2\right)$. See also Remark 14 for another definition of the classes $u_{2 k-1}$.

The following consequence follows immediately from Proposition 6 (b) and Remark 8.

Theorem 11. The isomorphism $\alpha: H^{*}\left(B G L(\mathbb{Z})^{+} ; \mathbb{Z} / 2\right) \stackrel{\cong}{\longrightarrow} H^{*}(B O ; \mathbb{Z} / 2) \otimes$ $H^{*}(S U ; \mathbb{Z} / 2)$ given by Theorem 1 is unique and satisfies $\alpha\left(u_{2 k-1}\right)=1 \otimes v_{2 k-1}$ for $k \geq 2$. Therefore, there is an isomorphism of $\mathcal{A}$-module Hopf algebras

$$
H^{*}\left(B G L(\mathbb{Z})^{+} ; \mathbb{Z} / 2\right) \cong \mathbb{Z} / 2\left[w_{1}, w_{2}, \ldots\right] \otimes \Lambda\left(u_{3}, u_{5}, \ldots\right) .
$$

It follows from Theorem 11 and Lemma 2 (a) that the action of the Steenrod algebra on the classes $u_{2 k-1}$ is described by the following Lemma 12. However, we mention here another proof, based on the definition of $\xi_{k}$, which provides an explicit computational argument for the existence of the isomorphism of $\mathcal{A}$-module Hopf algebras $\alpha$.

Lemma 12. For all $k \geq 2, S q^{2 i} u_{2 k-1}=\left(\begin{array}{c}k-1 \\ i\end{array}\right) u_{2 k+2 i-1}$ for $1 \leq i<k$ and $S q^{2 i-1} u_{2 k-1}=0$ for $1 \leq i \leq k$.

Proof. It is sufficient to prove the assertion for the classes $u_{2 k-1}(p)$ where $p$ is any prime $\equiv 5 \bmod 8$. This follows from the injectivity of the map $\rho$ which was explained just after the proof of Lemma 3 (if $m$ is large enough) and from the computations $S q^{2 i-1} \xi_{k}=0$ and

$$
S q^{2 i} \xi_{k}=\sum_{j=1}^{m} S q^{2 i} x_{j}^{k-1} y_{j}=\sum_{j=1}^{m}\left(\begin{array}{c}
k-1 \\
i
\end{array}\right) x_{j}^{k+i-1} y_{j}=\left(\begin{array}{c}
k-1 \\
i
\end{array}\right) \xi_{k+i} .
$$


It is even possible to describe the classes $u_{2 k-1}$ in terms of the image of $f_{p}^{*}$ for all primes $p \equiv 3$ or $5 \bmod 8$.

Theorem 13. For $k \geq 2$, the classes $u_{2 k-1} \in H^{2 k-1}\left(B G L(\mathbb{Z})^{+} ; \mathbb{Z} / 2\right)$ satisfy

$$
u_{2 k-1}= \begin{cases}f_{p}^{*}\left(e_{k}\right)+\sum_{j=1}^{k-2} w_{j}^{2} u_{2 k-2 j-1}, & \text { if } p \equiv 5 \bmod 8, \\ f_{p}^{*}\left(e_{k}\right)+w_{2 k-1}+\sum_{j=1}^{k-1} w_{j} w_{2 k-j-1}+\sum_{j=1}^{k-2} w_{j}^{2} u_{2 k-2 j-1}, & \text { if } p \equiv 3 \bmod 8\end{cases}
$$

where $f_{p}^{*}$ denotes the homomorphism $H^{*}\left(F \Psi^{p} ; \mathbb{Z} / 2\right) \cong H^{*}\left(B G L\left(\mathbb{F}_{p}\right)^{+} ; \mathbb{Z} / 2\right) \rightarrow$ $H^{*}\left(B G L(\mathbb{Z})^{+} ; \mathbb{Z} / 2\right)$ induced by the reduction $\bmod p: G L(\mathbb{Z}) \rightarrow G L\left(\mathbb{F}_{p}\right)$.

Proof. If $p \equiv 5 \bmod 8$, the statement is given by Proposition 6 (a). Observe in particular that $u_{2 k-1}$ can be written as follows: $u_{2 k-1}=F_{k}\left(f_{p}^{*}\left(e_{2}\right), f_{p}^{*}\left(e_{3}\right), \ldots, f_{p}^{*}\left(e_{k}\right)\right)$, where $F_{k}$ is a polynomial with coefficients in $\mathbb{Z} / 2\left[w_{1}, w_{2}, \ldots\right]$. If $p \equiv 3 \bmod 8$, consider again

$$
\gamma_{k}=f_{p}^{*}\left(e_{k}\right)+w_{2 k-1}+\sum_{j=1}^{k-1} w_{j} w_{2 k-j-1}
$$

and define $\widehat{u}_{2 k-1}=F_{k}\left(\gamma_{2}, \gamma_{3}, \ldots, \gamma_{k}\right)$. It is obvious that $\widehat{u}_{2 k-1}$ is an exterior class and easy to check as in the proof of Proposition 6 that $\eta^{*}\left(\widehat{u}_{2 k-1}\right)=v_{2 k-1}$. Moreover, observe that the homomorphism

$$
\mu^{*}: H^{*}\left(B G L(\mathbb{Z})^{+} ; \mathbb{Z} / 2\right) \rightarrow H^{*}\left(B G L(\mathbb{Z})^{+} \times B G L(\mathbb{Z})^{+} ; \mathbb{Z} / 2\right)
$$

acts on $\gamma_{k}$ (for $\left.p \equiv 3 \bmod 8\right)$ and on $f_{p}^{*}\left(e_{k}\right)$ (for $p \equiv 5 \bmod 8$ ) exactly in the same way, according to Lemma 3 (b) and (c). Thus, the argument of the proof of Proposition 7 implies that $\widehat{u}_{2 k-1}$ is also primitive if $p \equiv 3 \bmod 8$. It finally follows from Remark 8 that

$$
u_{2 k-1}=\widehat{u}_{2 k-1}=F_{k}\left(\gamma_{2}, \gamma_{3}, \ldots, \gamma_{k}\right)=\gamma_{k}+\sum_{j=1}^{k-2} w_{j}^{2} u_{2 k-2 j-1} .
$$

Remark 14. The formula provided by Theorem 13 can be used as an alternative recursive definition of the classes $u_{2 k-1}$ in $H^{*}\left(B G L(\mathbb{Z})^{+} ; \mathbb{Z} / 2\right)$.

It is known that $B G L(\mathbb{Z})^{+} \simeq B S L(\mathbb{Z})^{+} \times B \mathbb{Z} / 2$ and one deduces immediately the calculation of the mod 2 cohomology of the space $B S L(\mathbb{Z})^{+}$(recall that $H^{*}\left(B S L\left(\mathbb{F}_{p}\right)^{+} ; \mathbb{Z} / 2\right)$ is obtained from $H^{*}\left(B G L\left(\mathbb{F}_{p}\right)^{+} ; \mathbb{Z} / 2\right)$ by dividing out $e_{1}$ and $\left.c_{1}\right)$ :

Corollary 15. There is an isomorphism of $\mathcal{A}$-module Hopf algebras

$$
H^{*}\left(B S L(\mathbb{Z})^{+} ; \mathbb{Z} / 2\right) \cong \mathbb{Z} / 2\left[w_{2}, w_{3}, \ldots\right] \otimes \Lambda\left(u_{3}, u_{5}, \ldots\right),
$$

where $w_{k}$ and $u_{2 k-1}$ are also written for the image of $w_{k}$ and $u_{2 k-1}$ under the homomorphism induced by the inclusion $S L(\mathbb{Z}) \hookrightarrow G L(\mathbb{Z})$. The formulas for $u_{2 k-1}$ given by Theorem 13 do still hold but observe that the first Stiefel-Whitney class of $S L(\mathbb{Z})$ is trivial. 
Remark 16. The results of this section determine also the mod 2 cohomology of the groups $G L(\mathbb{Z})$ and $S L(\mathbb{Z})$ because $H^{*}\left(B G^{+} ; \mathbb{Z} / 2\right) \cong H^{*}(G ; \mathbb{Z} / 2)$ for $G=G L(\mathbb{Z})$ or $S L(\mathbb{Z})$.

\section{The mod 2 cohomology of the Steinberg group $\operatorname{St}(\mathbb{Z})$}

The goal of this last section is to compute $H^{*}(S t(\mathbb{Z}) ; \mathbb{Z} / 2)$ by looking at the universal central extension

$$
\mathbb{Z} / 2 \cong K_{2}(\mathbb{Z}) \succ S t(\mathbb{Z}) \stackrel{\pi}{\longrightarrow} S L(\mathbb{Z})
$$

and at the associated Serre spectral sequence

$$
E_{2}^{*, *} \cong H^{*}(S L(\mathbb{Z}) ; \mathbb{Z} / 2) \otimes H^{*}(\mathbb{Z} / 2 ; \mathbb{Z} / 2) \Longrightarrow H^{*}(S t(\mathbb{Z}) ; \mathbb{Z} / 2) .
$$

Let us use the notation $Q_{0}=S q^{1}$ and $Q_{r}=S q^{2^{r}} Q_{r-1}+Q_{r-1} S q^{2^{r}}$ and observe that $Q_{r}\left(w_{2}\right)=S q^{2^{r}} S q^{2^{r-1}} \cdots S q^{1} w_{2}$ because $S q^{2^{r}} w_{2}=0$ for $r \geq 2$ and $S q^{1} S q^{2} w_{2}=0$.

Theorem 17. (a) There is an isomorphism of $\mathcal{A}$-module Hopf algebras

$$
H^{*}(S t(\mathbb{Z}) ; \mathbb{Z} / 2) \cong \mathbb{Z} / 2\left[\bar{w}_{2}, \bar{w}_{3}, \ldots\right] /\left(\bar{w}_{2}, Q_{r}\left(\bar{w}_{2}\right), r \geq 0\right) \otimes \Lambda\left(\bar{u}_{3}, \bar{u}_{5}, \ldots\right),
$$

where $\bar{w}_{k}$ and $\bar{u}_{2 k-1}$ denote the image of $w_{k}$ and $u_{2 k-1}$ under $\pi^{*}: H^{*}(S L(\mathbb{Z}) ; \mathbb{Z} / 2)$ $\rightarrow H^{*}(S t(\mathbb{Z}) ; \mathbb{Z} / 2)$.

(b) For $k \geq 2$,

$$
\bar{u}_{2 k-1}= \begin{cases}f_{p}^{*}\left(e_{k}\right)+\sum_{j=4}^{k-2} \bar{w}_{j}^{2} \bar{u}_{2 k-2 j-1}, & \text { if } p \equiv 5 \bmod 8 \\ f_{p}^{*}\left(e_{k}\right)+\bar{w}_{2 k-1}+\sum_{j=4}^{k-1} \bar{w}_{j} \bar{w}_{2 k-j-1}+\sum_{j=4}^{k-2} \bar{w}_{j}^{2} \bar{u}_{2 k-2 j-1}, & \text { if } p \equiv 3 \bmod 8\end{cases}
$$

where $f_{p}^{*}$ is written here for the homomorphism $H^{*}\left(S L\left(\mathbb{F}_{p}\right) ; \mathbb{Z} / 2\right) \rightarrow H^{*}(S t(\mathbb{Z}) ; \mathbb{Z} / 2)$ induced by the reduction $\bmod p: S t(\mathbb{Z}) \rightarrow S t\left(\mathbb{F}_{p}\right) \cong S L\left(\mathbb{F}_{p}\right)$.

Proof. Because $H^{*}(\mathbb{Z} / 2 ; \mathbb{Z} / 2) \cong \mathbb{Z} / 2[z]$ with $\operatorname{deg} z=1$, one can compute the differentials in the above spectral sequence:

$$
d_{2}(z)=w_{2}, \quad d_{3}\left(z^{2}\right)=S q^{1} d_{2}(z)=S q^{1} w_{2}=w_{3}, \quad d_{5}\left(z^{4}\right)=S q^{2} d_{3}\left(z^{2}\right)=S q^{2} w_{3}
$$

and inductively, $d_{2^{r}+1}\left(z^{2^{r}}\right)=d_{2^{r}+1}\left(Q_{r-1}(z)\right)=Q_{r-1}\left(d_{2}(z)\right)=Q_{r-1}\left(w_{2}\right)=w_{2^{r}+1}$ + (decomposable element of $\mathbb{Z} / 2\left[w_{2}, w_{3}, \ldots\right]$ ) by Wu's formula ([MT], Part I, p. 141). Therefore, the sequence $\left(w_{2}, Q_{0}\left(w_{2}\right), Q_{1}\left(w_{2}\right), \ldots\right)$ is regular and we obtain $E_{\infty}^{s, t}=0$ if $t>0$ and $E_{\infty}^{*, 0} \cong H^{*}(S L(\mathbb{Z}) ; \mathbb{Z} / 2) /\left(w_{2}, Q_{r}\left(w_{2}\right), r \geq 0\right)$. This gives the mod 2 cohomology of $S t(\mathbb{Z})$ as described by statement (a) and assertion (b) follows directly from Theorem 13 and Corollary 15 since $\bar{w}_{2}=\bar{w}_{3}=0$.

Remark 18. The above argument exhibits a surjective homomorphism from $H^{*}\left(B S L(\mathbb{Z})^{+} ; \mathbb{Z} / 2\right)$ to $H^{*}\left(B S t(\mathbb{Z})^{+} ; \mathbb{Z} / 2\right)$. However, it is actually possible to find a nice map from $B S t(\mathbb{Z})^{+}$to the space $B S p i n$ inducing an injective homomorphism on mod 2 cohomology. More precisely, consider the map $\varepsilon: B S L(\mathbb{Z})^{+} \rightarrow B S L(\mathbb{R})^{+}$ induced by the inclusion $\mathbb{Z} \hookrightarrow \mathbb{R}$ and the map $\kappa: B S L(\mathbb{R})^{+} \rightarrow B S L(\mathbb{R})^{\text {top }} \simeq B S O$ induced by the obvious map $S L(\mathbb{R}) \rightarrow S L(\mathbb{R})^{\text {top }}$, where the first group $S L(\mathbb{R})$ is 
endowed with the discrete topology and $S L(\mathbb{R})^{\text {top }}$ with the usual topology. Then, look at the commutative diagram

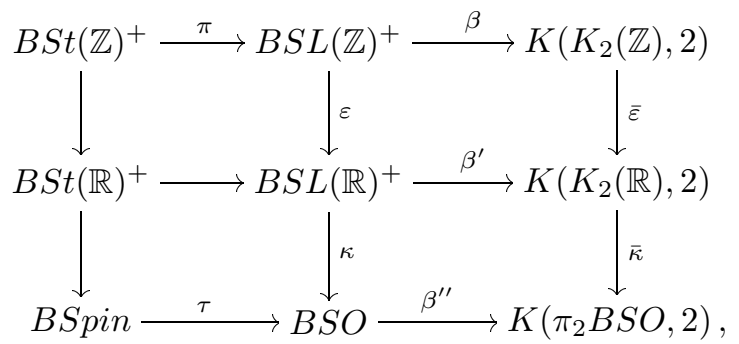

where the rows are fibrations in which the maps $\beta, \beta^{\prime}, \beta^{\prime \prime}$ are the second Postnikov sections of the corresponding spaces (BSpin is the fiber of $\beta^{\prime \prime}$ ), the maps $\bar{\varepsilon}$ and $\bar{\kappa}$ are the second Postnikov sections of $\varepsilon$ and $\kappa$, and the vertical maps on the left are the restrictions of $\varepsilon$ and $\kappa$ to the fibers. The composition $\bar{\kappa} \bar{\varepsilon}$ is a homotopy equivalence because $\bar{\kappa}_{*} \bar{\varepsilon}_{*}: K_{2}(\mathbb{Z}) \rightarrow \pi_{2} B S O$ is an isomorphism (see Corollary 4.6 of $[\mathrm{Br}]$ or p. $25-26$ of [Be]). Let us denote the composition $\kappa \varepsilon$ by $\chi$ and its restriction to $B S t(\mathbb{Z})^{+}$by $\tilde{\chi}: B S t(\mathbb{Z})^{+} \rightarrow B S p i n$ (note that the 2-completion of $\chi$ is the universal cover of the map $h$ defined in the introduction and that the fiber of the 2-completion of $\tilde{\chi}$ is $S U_{2}$ because of the diagram $\left.(*)\right)$. We get the commutative diagram

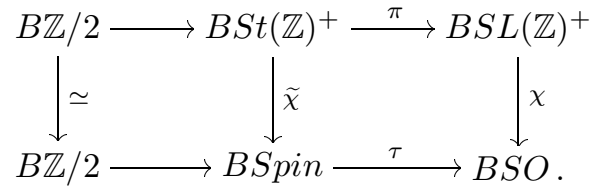

The ring structure of the mod 2 cohomology of BSpin is known by Proposition 6.5 of [Q1]:

$$
H^{*}(B \operatorname{Spin} ; \mathbb{Z} / 2) \cong \mathbb{Z} / 2\left[\widetilde{w}_{2}, \widetilde{w}_{3}, \ldots\right] /\left(\widetilde{w}_{2}, Q_{r}\left(\widetilde{w}_{2}\right), r \geq 0\right),
$$

where the $\widetilde{w}_{k}$ 's are written here for the image of the universal Stiefel-Whitney classes under the homomorphism $\tau^{*}: H^{*}(B S O ; \mathbb{Z} / 2) \rightarrow H^{*}(B \operatorname{Spin} ; \mathbb{Z} / 2)$. Since $\chi^{*}$ : $H^{*}(B S O ; \mathbb{Z} / 2) \rightarrow H^{*}\left(B S L(\mathbb{Z})^{+} ; \mathbb{Z} / 2\right)$ is injective, the map $\tilde{\chi}$ induces an injective $\mathcal{A}$-module Hopf algebra homomorphism

$$
\tilde{\chi}^{*}: H^{*}(B \operatorname{Spin} ; \mathbb{Z} / 2) \rightarrow H^{*}\left(B S t(\mathbb{Z})^{+} ; \mathbb{Z} / 2\right) .
$$

\section{REFERENCES}

[Ar1] D. Arlettaz: Torsion classes in the cohomology of congruence subgroups, Math. Proc. Cambridge Philos. Soc. 105 (1989), 241-248. MR 90j:20097

[Ar2] D. Arlettaz: A note on the mod 2 cohomology of $S L(\mathbb{Z})$, in: Algebraic Topology Poznań 1989, Proceedings, Lecture Notes in Math. 1474 (1991), 365-370. MR 93g:19005

[Au] C. Ausoni: Propriétés homotopiques de la K-théorie algébrique des entiers, Ph.D. thesis, Université de Lausanne (1998).

[Be] J. Berrick: An Approach to algebraic K-theory. (Pitman, 1982). MR 84g:18028

[Bok] M. Bökstedt: The rational homotopy type of $\Omega \mathrm{Wh}^{\text {Diff }}(*)$, in: Algebraic Topology, Aarhus 1982, Lecture Notes in Math. 1051 (1984), 25-37. MR 86e:18011

[Bor] A. Borel: Topics in the homology theory of fibre bundles, Lecture Notes in Math. 36 (1967). MR 36: 4559

[Br] W. Browder: Algebraic K-theory with coefficients $\mathbb{Z} / p$, in: Geometric Applications of Homotopy Theory I, Evanston 1977, Lecture Notes in Math. 657 (1978), 40-84. MR 80b:18011 
[DF] W. Dwyer and E. Friedlander: Conjectural calculations of general linear group homology, in Applications of Algebraic K-theory to Algebraic Geometry and Number Theory, Boulder 1983, Contemp. Math. 55 Part I (1986), 135-147. MR 88f:18013

[FP] Z. Fiedorowicz and S. Priddy: Homology of classical groups over finite fields and their associated infinite loop spaces, Lecture Notes in Math. 674 (1978). MR 80g:55018

[M] S. Mitchell: On the plus construction for $B G L \mathbb{Z}\left[\frac{1}{2}\right]$ at the prime 2, Math. Zeitschrift 209 (1992), 205-222. MR 93b:55021

[MT] M. Mimura and H. Toda: Topology of Lie groups I and II, Translations of Math. Monographs 91 (AMS 1991). MR 92h:55001

[Q1] D. Quillen: The mod 2 cohomology rings of extra-special 2-groups and spinor groups, Math. Ann. 194 (1971), 197-212. MR 44:7582

[Q2] D. Quillen: On the cohomology and K-theory of the general linear groups over a finite field, Ann. of Math. 96 (1972), 552-586. MR 47:3565

[RW] J. Rognes and C. Weibel: Two-primary algebraic K-theory of rings of integers in number fields, preprint (1997), http://math.uiuc.edu/K-theory/0220/.

[V] V. Voevodsky: The Milnor conjecture, preprint (1996), http://math.uiuc.edu/Ktheory $/ 0170 /$.

[W] C. Weibel: The 2-torsion in the K-theory of the integers, C. R. Acad. Sci. Paris Sér. I 324 (1996), 615-620. MR 98h:19001

Institut de Mathématiques, Université de Lausanne, 1015 Lausanne, Switzerland

E-mail address: dominique.arlettaz@ima.unil.ch

Department of Mathematics, Faculty of Science, Okayama University, Okayama, JAPAN 700

E-mail address: mimura@math.okayama-u.ac.jp

Institut de Mathématiques, Université de Lausanne, 1015 Lausanne, Switzerland

E-mail address: koji.nakahata@ima.unil.ch

Faculty of Educhtion, Ibaraki University, Mito, Ibaraki, Japan

E-mail address: yagita@mito.ipc.ibaraki.ac.jp 